THURSDAY, JUNE 29, I9II.

\section{DYNAMICAL, METEOROLOGY AND HYDROGRAPHY.}

Dynamic Meteorology and Hydrography. By Prof. V. Bjerknes and different collaborators. P. I46+ $3^{6 A}+3 \mathrm{OB}+22 \mathrm{C}$. (Washington, D.C. : Carnegie Institution, I9 Io.)

$\mathrm{N}$ a lecture delivered at University College in May, IgIo, Prof. Bjerknes outlined the methods, described characteristically as rational, by which he hoped to utilise synchronous meteorological observations for a more purely scientific purpose than the preparation of daily forecasts. The present work is the first instalment of a treatise prepared, in collaboration with Dr. J. W. Sandström, with such investigations in view, and is intended to present in an ordered and rational form the principles and development of meteorology and hydrography viewed from the point of view of a mathematical physicist. Bjerknes has realised the fact that economy: of thought and labour are essential to advance in meteorological and hydrographic investigation, and although his book bears little actual resemblance to Lagrange's "Mécanique Analytique," we cannot help calling to mind that classical masterpiece of scientific economy in reading this volume.

The existing chaos in meteorological units has led Bjerknes, among others, to the conclusion that a thorough reform in this respect will go far towards making meteorological progress possible through the practical application of more advanced mathematical treatment than is at present customary. He begins therefore by introducing as suitable units for meteorological purposes the metre, the metric ton, or $10^{6}$ grams, and the second, and calls this briefly the m.t.s. system. The metre and the ton are chosen on the ground that the centimetre and the gram are too small as units of length and mass for practical applications, in much the same way as the c.g.s. electrical units are in general unsuitable for the practical engineer. The atmosphere, however, resembles a thin plate, and although the vertical dimensions and motions are relatively small, they are nevertheless important. No combination of units will be appropriate for all cases, and Bjerlsnes himself departs in some cases from the rational derived units which follow from his scheme. It seems doubtful therefore if it is a wise plan to run the risk of discouraging the reader at the outset by the formal introduction of new units.

The first two chapters are introductory in character, and deal with the units used in the work and with gravity and the corresponding scalar, gravity potential. The unit of gravity potential on the m.t.s. system is called the dynamic decimetre, because it is equal to the work done in lifting unit mass against gravity through a height which is approximately equal to a decimetre. For practical application, however, the dynamic metre is taken, and this unit is fundamental in Bjerlknes's work. Its great advantage is that the distance to which it corresponds agrees sufficiently NO. 2 I 74 , VOL. 86$]$ closely with the metre, to make it suitable for expressing approximately geometric heights, and points at the same distance in dynamic metres from the earth's surface (sea-level) are on the same level surface of gravity. Bjerknes even makes out a case for publishing the results of geodetic determinations of "height" in dynamic metres, just as in a later chapter he emphasises the need for giving the corresponding values of pressure and temperature in the publication of the results of upper-air observations, instead of height and temperature, the quantities more frequently adopted at present.

Some care is needed to prevent confusion in connection with dynamic decimetres and metres. These are units of work and are invariable, but the heights, with which they correspond, vary with the locality inversely as the value of gravity.

The m.t.s. unit of pressure is the centibar, but here again it is found convenient to take as the practical unit the bar or the megadyne per square centimetre, and the graduation of the barometer in " millibars" is advocated. It seems necessary to proceed cautiously in this connection. It is proper and scientific to express atmospheric pressure in terms of the megadyne per square centimetre or the bar, and it is legitimate to take advantage of any practical device which will enable this to be done as easily as possible; but in using the mercury barometer we are primarily measuring a distance, and equal increments of height do not correspond with equal increments of pressure at different places, or under different conditions at the same place. Meteorologists ought to beware of adding another incongruity to the list of those which they ridicule frequently in a good-humoured way when they have become accustomed to the feeling that long usage has made the bonds too strong to be broken.

Chapter iii. deals with the specific volume and density of air and sea-water. Owing to the fact that the amount of water-vapour present in the atmosphere is a variable quantity, the "constant," $R$, in the equation $p v=\mathrm{RT}$ is variable also, and this constitutes a real difficulty in the discussion of atmospheric changes. Bjerknes reduces the difficulty very considerably by adopting the artifice of keeping $R$ constant throughout, and using in the equation, not the actual temperature $\mathrm{T}$, but the virtual temperature $\tau$, which is the temperature at which dry air would have the same density as the air under consideration. By another ingenious device he makes seven small tables for obtaining the density of sea-water from the temperature, salinity and pressure cover the same range as a quarter of a million pages of straightforward tabulation.

The next two chapters are concerned with the principles of hydrostatics and their application to the atmosphere in the case of constant temperature gradient and for adiabatic equilibrium. They contain an instructive set of diagrams showing for the same scale of height (measured in dynamic metres) the pressure, density, and specific volume of the atmosphere at different levels for the four cases, homogeneous atmosphere, dry atmosphere in adiabatic equilibrium, atmosphere with constant vertical 
gradient of temperature $0^{\circ} 5^{\circ} \mathrm{C}$. per $100 \mathrm{~m}$., and iso thermal atmosphere. Thus it is interesting to observe that at $20,000 \mathrm{~m}$., for example, the pressures expressed in millibars are in the four cases $0,10,42,79$ respectively.

Chapter vi. is devoted to a consideration of the problem of determining the heights at given pressures or the pressures at given heights when the virtual temperatures at given pressures or at given heights are known. The method developed is applied to particular cases in which the observed quantities have been found from the records obtained by means of registering balloons. In calculating the height at which a given isobaric surface is to be found, the distances between consecutive isobaric surfaces are taken directly from the tables, so that the single process gives the entire representation of the field of pressure and mass. The ease and simplicity of the method suggest that meteorologists may find it preferable in their synchronous charts to represent the heights at which a standard isobaric surface is to be found instead of the pressures at a standard level. In chapter vii. such charts are drawn for different isobaric surfaces by using the results of the international ascents of registering balloons. Profile diagrams are also drawn showing the section, by a vertical plane, of the isobaric and isosteric (constant specific volumel surfaces; and of the equipotential and isopycnic (constant density) surfaces. In the equilibrium state no two of these surfaces intersect, and the number of tubes made by their intersections in actual cases is a measure of the departure from the equilibrium condition. This method of viewing the distribution is very suggestive, and deserves further development and application.

Chapters viii. and ix. are hydrographic counterparts of chapters vi. and vii., and complete the formal development presented in this volume, the remaining eighty pages being devoted to the tables necessary for the application of Bjerknes's methods. They will be found of great use in the discussion of the atmosphere as a fluid in three dimensions. They differ in some respects from the tables constructed five years ago by Sandstrom, and they cover a wider field. There are slight differences in the values for the distance between consecutive isobaric surfaces, which are no doubt due to the revision of Sandström's results.

The impression produced by a study of Bjerknes's book is that it does not contain new discoveries or throw much fresh light on individual atmospheric phenomena, but it presents what is fundamental in our knowledge of the physics of the atmosphere in a new way, and makes possible the application of methods which have hitherto been disregarded, because of the immense labour involved in dealing with even a single case. The temptation to pad the work with examples has been successfully resisted, and the cases discussed are confined to what is strictly necessary in the scheme of development.

The observations in the upper air obtained by means of kites and balloons have hitherto been little used in the synoptic representation of atmospheric conditions, and in the investigation of the dynamical NO. 2 I 74, VOL. 867 problems which a three-dimensional knowledge was expected to elucidate. They have indeed achieved much in giving us definite lenowledge in place of erroneous hypotheses, but ambitious minds naturally wish to turn them to practical use in daily forecasting. Laborious investigation is an essential preliminary to such an application, and Bjerknes, with his large, enthusiastic spirit, has taken up this work in a way which ought to secure him the active support of professional meteorologists. The book is excellently printed, and its form and style produce a sense of pleasure and satisfaction. There is an old rule about leaving the preface of a book until the remainder is completed. Apparently Bjerlknes is talking this rule so literally that he is reserving the introduction, as well as the index, until the issue of the final volume.

E. GOLD.

\section{THE JEWS}

The Jews: a Study of Race and Environment. By M. Fishberg. Pp. xix +598 . (London and Fellingon-Tyne: Walter Scott Publishing Co., Ltd., IgII.) Price $6 s$.

I $\mathrm{N}$ order to elucidate the problem whether the Jews constitute a race or simply a nation, Mr. Fishberg discusses at considerable length certain physical characters, with the following results. Stature is not homogeneous among the Jews in every country, and its limits of variation are almost as large as are observed in European races generally; further, where the indigenous population is tall the Jews are also tall, and the reverse. It is also evident that the shortness of their stature can be attributed only to a slight extent to the influence of environment or to occupation. Jewish skulls are extremely rare in museums; indeed, there do not appear to be any data whatever for the ancient Hebrews; the cranial index of five skulls of the second century, found in Rome, varies from $75^{\circ}$ to $83^{\circ} 4$; of twelve skulls found in Basel in a cemetery dating from the thirteenth and fourteenth centuries two were dolichocephalic, while the remainder were brachycephalic, the total average being $84^{\circ} 6$. The same variability occurs in other finds, but the skulls of most of the Sephardim-or Spanish and Portuguese Jews-are dolichocephalic.

Among the existing population it is found that in countries where the indigenous population is narrowheaded, as in Africa and Arabia, the Jews are dolichocephalic, and where broad-headedness prevails, as in Russia, Poland, and Hungary, the Jews are brachycephalic. The ancient Hebrews must have been either of the one type or the other, or a mixed race originally; the former alternative implies that for most of the Jews miscegenation must have occurred in later times. Although predominantly dark, fair Jews are found everywhere; even among the Sephardim, it appears that the blond type oscillates between 5 and 16 per cent., and between 25 and 50 per cent. are of the mixed types according to the country of birth. The suggestion that the blondness is a product of climatic conditions can be eliminated as worthless, as blond Jews occur everywhere. The combination of tall stature, blond- 